\title{
An Inhomogeneous Bayesian Texture Model for Spatially Varying Parameter Estimation
}

\author{
Chathurika Dharmagunawardhana ${ }^{1}$, Sasan Mahmoodi ${ }^{1}$, Michael Bennett $^{2}$ and Mahesan Niranjan ${ }^{1}$ \\ ${ }^{1}$ School of Electronics and Computer Science, University of Southampton, Southampton, SO17 1BJ, U.K. \\ ${ }^{2}$ National Institute for Health Research, Southampton Respiratory Biomedical Research Unit, \\ University Hospital Southampton NHS Foundation Trust, Tremona Road, Southampton, U.K. \\ $\{c d 6 g 10$, sm3,mn\}@ecs.soton.ac.uk,michaelbennett@soton.ac.uk
}

Keywords: $\quad$ Spatially Varying Parameters, Gaussian Markov Random Fields, Bayesian Modeling, Texture Classification, Texture Segmentation.

\begin{abstract}
In statistical model based texture feature extraction, features based on spatially varying parameters achieve higher discriminative performances compared to spatially constant parameters. In this paper we formulate a novel Bayesian framework which achieves texture characterization by spatially varying parameters based on Gaussian Markov random fields. The parameter estimation is carried out by Metropolis-Hastings algorithm. The distributions of estimated spatially varying parameters are then used as successful discriminant texture features in classification and segmentation. Results show that novel features outperform traditional Gaussian Markov random field texture features which use spatially constant parameters. These features capture both pixel spatial dependencies and structural properties of a texture giving improved texture features for effective texture classification and segmentation.
\end{abstract}

\section{INTRODUCTION}

Markov Random Fields (MRF) have been successfully used in texture modeling and well recognized in the field of texture synthesis, classification and segmentation. The Gaussian MRF (GMRF) is a special case of MRFs which has less computational cost involved with its parameter estimation (Li, 2009). Model parameters of the GMRF offer descriptive features for texture analysis and have been directly used in texture classification and segmentation (Manjunath and Chellappa, 1991; Chellappa and Chatterjee, 1985). It is referred to as the traditional GMRF (TGMRF) feature extraction method and the parameter estimation is performed either by least square estimation (LSE) or maximum likelihood estimation (MLE) (Zhao et al., 2007; Mahmoodi and Gunn, 2011; Dharmagunawardhana et al., 2012).

Techniques have been proposed to enhance the discriminative power of TGMRF features. These methods specially focus on either improving the parameter estimation process of TGMRF method or finding systematic ways of selecting factors involving the estimation process, for example neighborhood size (Zhao et al., 2007).

However, the previous studies on texture feature extraction using GMRFs are focused only on spatially constant model parameters. A texture is assumed to be a stationary random field having a unique spatially constant model parameter set characterizing it (Manjunath and Chellappa, 1991; Zhao et al., 2007). A recent study on statistical model based feature extraction by (Dharmagunawardhana et al., 2012) have emphasized the significance of using spatially varying parameters, with the intention of achieving more discriminative features rather than exact modeling of the texture. The spatially varying parameter estimation process in (Dharmagunawardhana et al., 2012) is based on a simple technique called small model estimation.

In the present study, our contribution is introducing a fully developed Bayesian framework to estimate spatially varying model parameters with MetropolisHastings algorithm. The Bayesian formulation allows integrating prior knowledge about the parameters to the estimation process. The current study uses smoothing priors to locally smooth the spatially varying parameter space. Therefore this approach can reduce the noise present in the spatially varying parameters while preserving the discriminative ability of the features formulated based on them. Our Bayesian framework for spatially varying parameter estimation 
is inspired by the inhomogeneous Bayesian model discussed in (Aykroyd, 1998) for image reconstruction. However, our study is different from theirs because our objective is constructing an inhomogeneous Bayesian model for texture.

After formulating the inhomogeneous texture model, the Metropolis-Hastings algorithm is used to estimate the spatially varying parameters. The distributions of spatially varying parameters are then constructed using normalized histograms and proposed as the texture features. The experimental results show that this approach can produce more discriminative texture features compared to spatially constant parameter estimation (Manjunath and Chellappa, 1991) and the small model estimation (Dharmagunawardhana et al., 2012). Furthermore, we have applied these features in supervised texture segmentation to extract regions of a given texture.

The remainder of this paper is organized as follows. Section 2 introduces the Bayesian framework employing the spatially constant and spatially varying parameters and explains the parameter estimation. In section 3 results and discussions are elaborated and finally, in section 4 the conclusions are given.

\section{BAYESIAN TEXTURE MODEL}

In this section we introduce the homogeneous texture model which is subsequently extended to formulate inhomogeneous model for texture. The main difference between the two models is that homogeneous texture model is defined by spatially constant parameters and the inhomogeneous model is described by spatially varying parameters.

\subsection{Homogeneous Texture Model}

Let a stationary random field of a texture on an image region $\Omega$ be represented by $Y . y_{i}$ represents the pixel value at a site $i$ and $i$ is the column wise linear index. The local conditional model of GMRF describes the relationship between a pixel and its neighbors $y_{j}$ on a neighborhood $j \in \tilde{N}_{i}$ using a Gaussian functional form and is given by,

$$
\begin{aligned}
& p\left(y_{i} \mid y_{j}, \boldsymbol{\alpha}, \boldsymbol{\sigma}, j \in \tilde{N}_{i}\right)= \\
& \frac{1}{\sqrt{2 \pi \sigma^{2}}} \exp \left\{-\frac{1}{2 \sigma^{2}}\left(y_{i}-\sum_{j \in \tilde{N}_{i}} \alpha_{j} \bar{y}_{j}\right)^{2}\right\}
\end{aligned}
$$

The $\boldsymbol{\alpha}=\left[\alpha_{j} \mid j=1 \ldots R\right]^{T}$ are the interaction coefficients which measure the influence by a neighbor intensity value at the neighbor position $j$ (Petrou and
Sevilla, 2006; Li, 2009). $R$ is the number of interaction parameters. The neighbor pixels in symmetric positions about the considered pixel are assumed to have identical parameters (Petrou and Sevilla, 2006), therefore $\bar{y}_{j}$ is the sum of two neighbor values situated in symmetric neighbor positions with respect to the pixel.

Assuming the conditional independence of pixel value given its neighbors, the joint distribution can be written as,

$$
\begin{aligned}
& p(Y \mid \mathbf{x})= \\
& \prod_{i} \frac{1}{\sqrt{2 \pi \sigma^{2}}} \exp \left\{-\frac{1}{2 \sigma^{2}}\left(y_{i}-\sum_{j \in \tilde{N}_{i}} \alpha_{j} \bar{y}_{j}\right)^{2}\right\}
\end{aligned}
$$

where $\mathbf{x}=[\boldsymbol{\alpha}, \boldsymbol{\sigma}]^{T}$ is the parameter vector of the model. This will be referred to as the homogeneous model of the texture and it also represent the likelihood of having the texture $Y$ given the GMRF parameter vector. The model parameters of the above model do not depend on the location. Therefore one unique set of parameters will characterize the texture. These spatially constant model parameters are unable to capture the spatial variations in parameters (Dharmagunawardhana et al., 2012). The homogeneous model therefore needs to be modified to describe spatial variance of parameters. The solution is to formulate the inhomogeneous model for texture.

\subsection{Inhomogeneous Model}

The inhomogeneous model is characterized by spatially varying model parameters instead of constant parameters. The objective of using spatially varying parameters is that they can capture the pixel interaction variations acting on the texture primitives (Dharmagunawardhana et al., 2012). Spatially varying parameter estimation is therefore able to capture the local inhomogeneities in the texture primitive and its arrangement patterns.

To obtain spatial variations in parameter space, a separate vector of model parameters for each pixel is defined. In this way, every pixel has its own vector of parameters. Let the parameter vector for pixel at site $i$ be $\mathbf{x}_{i}=\left[x_{i}^{j} \mid j=1, \ldots, R+1\right]$. Note that superscript index $j$ where $j=1, \ldots, R$ represents the type of model parameter according to neighbor position and $j=R+1$ represents the index to the variance parameter. The linear index $i$ represents the location of the pixel similar to section 2.1. Hence for every parameter type there will be a corresponding parameter image, $X^{j}, j=1, \ldots, R+1$, in spatial domain.

Figure 1 shows an example to clearly understand the parameters of the model. Here the first order 


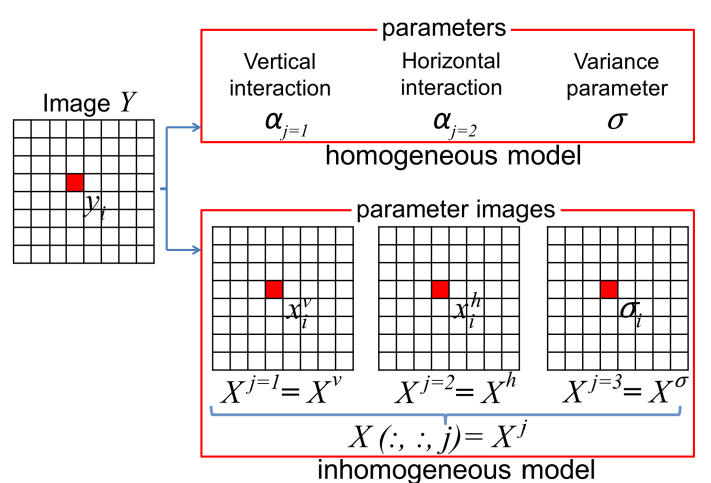

Figure 1: Parameters associated with the GMRF models.

neighborhood system is considered. Therefore three types of model parameters are involved in characterizing the model, namely, horizontal and vertical interaction parameters and the variance parameter. In the inhomogeneous model, for each type of parameter, for example the vertical interaction parameter, the parameter image is given by $X^{v}$ which represent the vertical interaction parameter values $x_{i}^{v}$ at each pixel $i \in \Omega$ of the spatial domain.

Once the parameters are defined and are assumed to be known, then the likelihood of the texture image $Y$ can be written as,

$$
\begin{aligned}
& p(Y \mid X)= \\
& \prod_{i} \frac{1}{\sqrt{2 \pi \sigma_{i}^{2}}} \exp \left\{-\frac{1}{2 \sigma_{i}^{2}}\left(y_{i}-\sum_{j \in \tilde{N}_{i}} x_{i}^{j} \bar{y}_{j}\right)^{2}\right\}
\end{aligned}
$$

Here the formulation of the inhomogeneous model involves many model parameters compared to spatially constant model formulation. But the estimation process can be easily parallelized using the coding scheme (Petrou and Sevilla, 2006) for a much faster estimation process.

The model parameter values on a parameter image will be repetitive according to the pattern repetition. Therefore the unique model parameter values on the parameter image correspond to the parameter values on one texel element. However, the size of the texel is not clearly identifiable in many types of textures, therefore we have considered a region of the texture, $\Omega$ as above. Then the distribution of the repetitive model parameters can be used to formulate the texture features.

Next we look at the prior distribution of the model. The prior model on parameters can also be defined as a GMRF on the parameter space. Here we limit our focus to smoothing priors. Alternately, any prior knowledge available on the location dependence of parameters could be associated to the prior model.
The prior model for interaction parameters can be written as,

$$
\begin{aligned}
& p\left(X^{j} \mid \gamma\right)= \\
& \prod_{i} \frac{1}{\sqrt{2 \pi \gamma^{2}}} \exp \left\{-\frac{1}{2 \gamma^{2}}\left(x_{i}^{j}-\frac{1}{\left|N_{i}\right|} \sum_{r \in N_{i}} x_{r}^{j}\right)^{2}\right\}
\end{aligned}
$$

$N_{i}$ is the neighbors of site $i$ on the parameter image $X^{j} .\left|N_{i}\right|$ is the number of neighbors. The above prior model is defined for $j=1, \ldots, R$. i.e. for interaction parameters. $\gamma^{2}$ is the hyper variance parameter of spatially varying model parameters. It is considered that the value of $\gamma$ is same for any interaction parameter $X^{j}$ for $j=1, \ldots, R$. The prior model for the variance parameter is,

$$
\begin{aligned}
& p\left(X^{R+1} \mid \delta\right)= \\
& \prod_{i} \frac{1}{\sqrt{2 \pi \delta^{2}}} \exp \left\{-\frac{1}{2 \delta^{2}}\left(\sigma_{i}-\frac{1}{\left|N_{i}\right|} \sum_{r \in N_{i}} \sigma_{r}\right)^{2}\right\}
\end{aligned}
$$

where $\delta$ is a constant and $\sigma_{i}$ is same as $x_{i}^{R+1}$ and is used for better readability (figure 1).

\subsection{Bayesian Formulation}

The posterior density for the inhomogeneous texture model can be written as follows.

$$
\begin{aligned}
p(X, \gamma, \delta \mid Y) & =\frac{p(X, \gamma, \delta, Y)}{p(Y)} \\
& =\frac{p(Y \mid X, \gamma, \delta) p(X \mid \gamma, \delta) p(\gamma, \delta)}{p(Y)}
\end{aligned}
$$

We assume the conditional independence between various variables to simplify the above expression, including the independence between hyper parameters, $\gamma$ and $\delta$. The posterior density can be then written as,

$$
\begin{array}{r}
p(X, \gamma, \delta \mid Y)=\frac{p(Y \mid X) p(X \mid \gamma, \delta) p(\gamma) p(\delta)}{p(Y)} \\
=\frac{p(Y \mid X) \prod_{j=1}^{R} p\left(X^{j} \mid \gamma\right) p\left(X^{R+1} \mid \delta\right) p(\gamma) p(\delta)}{p(Y)}
\end{array}
$$

Since there is no prior knowledge about $\gamma$ and $\delta$ uniform distributions for $p(\gamma)$ and $p(\delta)$ are assumed. All the other densities are defined as in section 2.2.

It is important to mention about the local conditional models of the joint models in (3), (4) and (5). A local conditional model tells us about how a pixel depends on its neighbors. Even though we use the global models in MCMC estimation, when calculating acceptance probabilities, all the terms will cancel out, except terms associated to the local models due 
to Markovian property given a symmetric proposal distribution. The local conditional models of (3), (4) and (5) intuitively become the expressions without the product symbol. But in this study we use a slightly altered local model for (3) as below.

$$
\begin{aligned}
& p\left(y_{i} \mid X_{i}\right)= \\
& \prod_{r}^{m} \frac{1}{\sqrt{2 \pi \sigma_{i}^{2}}} \exp \left\{-\frac{1}{2 \sigma_{i}^{2}}\left(y_{r}-\sum_{j \in \tilde{N}_{r}} x_{i}^{j} \bar{y}_{j}\right)^{2}\right\}
\end{aligned}
$$

$m$ represents the number of immediate neighbors of site $i$, for example eight neighbors around $i$. This expresses that probability of a pixel value not only depends on its neighborhood but also on near by $m$ pixels and their neighbors. It is a localized version of the likelihood of $m$ samples. Therefore $\sigma_{i}^{2}$ represents the variance considering $m$ local samples which is a localized variation at site $i$. Prior to using $m$ local samples their sample mean is set to zero.

\subsection{MCMC estimation}

Following (Aykroyd, 1998) we also use the Metropolis-Hastings (MH) algorithm for parameter estimation. Therefore finding normalizing constant of posterior distribution in (6) is no longer needed. Coding scheme (Petrou and Sevilla, 2006) is used to estimate parameters in parallel on each $X^{j}, j=$ $1, \ldots, R+1$. Each type of parameters including $\gamma$ and $\delta$ are sequentially estimated in turns. Approach to the estimation of various groups of model parameters is the same.

Let model parameters be represented by $\Theta$ where $\Theta=\{X, \gamma, \delta\}$. Let the parameter being considered be $\theta_{i}$. A proposed new value is selected from the proposal distribution $q\left(\theta_{i}^{\prime} \mid \theta_{i}\right)$. The set of parameters containing the proposed value is given by $\Theta^{\prime}=$ $\left\{\theta_{1}, \ldots, \theta_{i-1}, \theta_{i}^{\prime}, \theta_{i+1}, \ldots, \theta_{|\Omega|(R+1)+2}\right\}$. The proposed value of parameter is accepted and then updated with the acceptance probability,

$$
\min \left\{1, \frac{p\left(\Theta^{\prime} \mid Y\right) q\left(\theta_{i}^{\prime} \mid \theta_{i}\right)}{p(\Theta \mid Y) q\left(\theta_{i} \mid \theta_{i}^{\prime}\right)}\right\}
$$

Otherwise it is rejected and the previous value is retained. Doubly exponential distribution centered on the current value is used as the proposal distribution. The scale parameter of proposal distribution is chosen by trial and error technique. Since the proposal distribution is symmetric the ratio $q\left(\theta_{i}^{\prime} \mid \theta_{i}\right) / q\left(\theta_{i} \mid \theta_{i}^{\prime}\right)$ in (8) is canceled out. The values of $X^{R+1}, \gamma$ and $\delta$ are chosen to be positive all the time.

Many terms of the ratio in (8) will cancel out due to Markovian property. This leads to vastly simplified expressions. To avoid numerical overflow log value of posterior ratio is used. The Markov chain is developed with the accepted samples chosen according to the acceptance probability. The convergence of the chain is monitored graphically. When the chain is converged the average of samples laying outside the burn-in period are used as the expected value of the corresponding parameter.

Once the model parameters are estimated in this way, their spatial distributions constructed by normalized histograms can be used to formulate discriminative texture features.

\section{RESULTS AND DISCUSSION}

The Bayesian framework for the textures proposed here can be used to extract spatially varying model parameters and their spatial distributions can be used as effective texture features for classification.

In this paper, the focus is limited to the first order neighborhood system of GMRFs. Therefore three different types of model parameters, namely horizontal interaction parameter, vertical interaction parameter and variance parameter characterize the model.

Spatially varying model parameters are estimated by sampling the proposed posterior probability distribution in (6) and then taking the expected values of the samples excluding the burn-in period. MH algorithm is performed on each individual site to sample and estimate the parameters at that location. The coding scheme (Petrou and Sevilla, 2006) is used to speed up the process where instead of visiting each site in the image sequentially, a batch of pixels belonging to the same code is updated in parallel.

Each Markov chain is run for 2000 iterations. The first 500 samples are considered as the burn-in period. Rest of the samples are used to calculate the expected value of the parameter.

The scale parameters of doubly exponential proposal distributions are set by trial and error method for each type of model parameters. For Markov chain updates of interaction parameters, $X^{j}, j=1, \ldots, R$ the scale parameter is 0.05 and for the variance parameter $X^{R+1}$ it is 0.1 . For super parameters $\gamma$ and $\delta, 0.05$ and 0.1 are used respectively. The number of local samples for the likelihood model, $m$ is restricted to the five nearest samples in the proximity of considered site. This parameter setting is kept constant for all the experiments unless stated otherwise.

In figure 2 examples of estimated parameter images (expected values) achieved using the inhomogeneous Bayesian framework are shown. Figure 2a has two texture regions and corresponding horizon- 

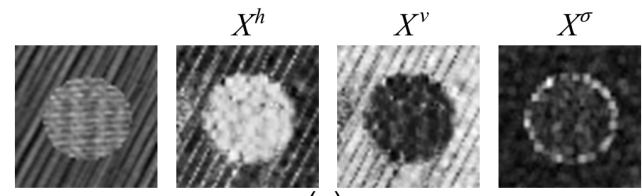

(a)
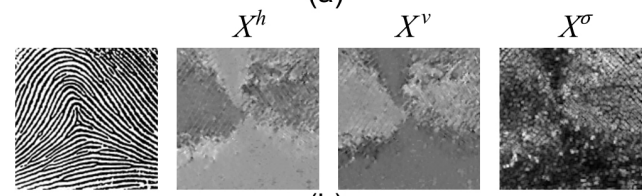

(b)

Figure 2: Parameter images obtained by inhomogeneous Bayesian framework for parameter estimation. $X^{h}-$ horizontal interaction parameter, $X^{v}$ - vertical interaction parameter, $X^{\sigma}$ - variance parameter

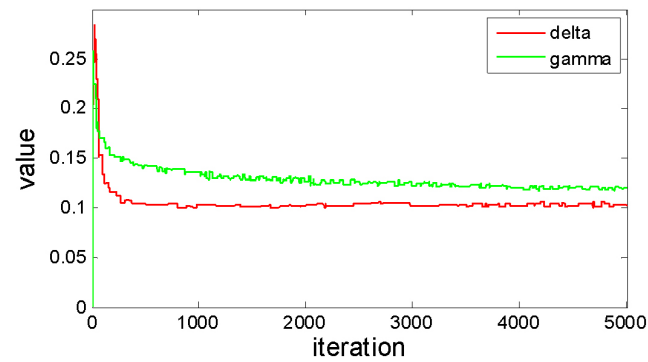

Figure 3: Markov Chains of $\delta$ and $\gamma$ parameters for image in figure $2 \mathrm{a}$.

tal and vertical interaction parameter images illustrate the spatially varying nature of the estimated model parameters. The variance parameter clearly indicates a higher variance near the boundary between the two texture regions. The pattern inside the circular region in figure 2a has more noteworthy horizontal interactions. The corresponding horizontal interaction parameter image has higher interaction parameter values in respective region. Also the pattern outside the circular region has a directional pattern closer to the vertical axis. Hence the corresponding vertical interaction parameter has higher interaction values outside the circular region.

Figure $2 b$ shows a partial finger print. The spatially varying parameters are not made rotational or scale invariant here. Therefore the corresponding spatially varying parameters capture the directional differences in the patterns of the finger print. In general, by looking at figure 2 it can be concluded that spatially varying model parameters carry more information about the texture.

The Markov chains of super parameters $\gamma$ and $\delta$ of figure $2 \mathrm{a}$ are shown in figure 3 . These chains graphically indicate the convergence roughly after 200 iterations. Therefore the burn-in period and number of iterations mentioned earlier are suitable values.

The local distributions of these spatially varying parameters can be used to discriminate texture regions. An evaluation of spatial distributions of spatially varying parameters is illustrated in figure 4 . Four textures are used and their corresponding spatially varying parameter images are obtained and converted into normalized histograms with 50 bins. The parameter image values are modified to lie between the range 0 to 255 before constructing the histograms. This normalization is done for illustration purposes only. The intensity histograms of the four textures are given in figure $4 \mathrm{c}$.

According to figure $4 \mathrm{c}$ it can be seen that discrimination power of the intensity histogram is quite low for these four textures. But the histograms of parameter images represent substantial differences in there distributions. The interaction parameter histograms in figure $4 \mathrm{~d}$ and e show a negative correlation between the distributions for a texture. Here we have only two interaction parameters in the model. Therefore, when the horizontal interaction is dominant, vertical interaction of the respective texture is much insignificant. However, these distributions of spatially varying model parameters can be used as a discriminative texture feature in texture analysis.

\subsection{General Texture Classification}

We perform texture classification using two datasets namely BRODATZ, a random subset of Brodatz dataset (Valkealahti and Oja, 1998) comprising 32 Brodatz textures (Brodatz, 1996) and OUTEX, the full OUTEX_TC_00001 dataset having 24 OUTEX textures (Ojala et al., 2002). Each dataset has 20 samples per class. Prior to feature extraction all the images are pre-processed using histogram equalization. Next, parameter estimation is carried out and the distributions of spatially varying parameters are constructed by normalized histograms.

The classification experiments are performed using equal sizes of training and test datasets randomly partitioned to have equal class proportions. The experiment is repeated 100 times with different training and test sets. Accuracies reported here are the mean accuracy of 100 iterations and its standard deviation. Classification is performed using nearest neighbor classifier with absolute difference distance metric.

The accuracies are given in table 1 . The proposed inhomogeneous model based feature extraction is referred to as $I B M F$ which stands for 'Inhomogeneous Bayesian Model based Features'. The feature extraction based on homogeneous Bayesian model is labeled as $H B M F$ which stands for 'homogeneous Bayesian Model based Features'. Four other methods have been used for performance compari- 


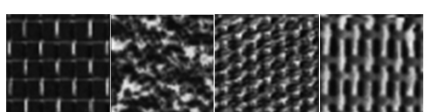

(a)

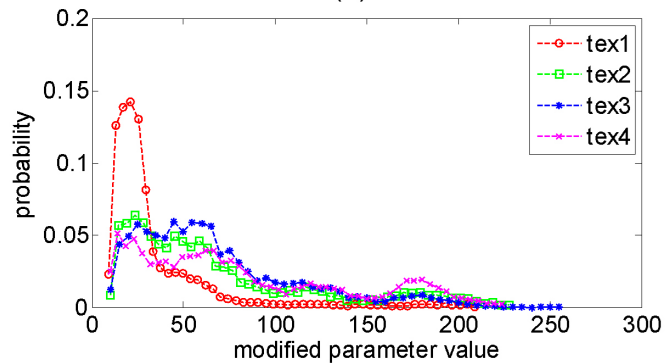

(c)

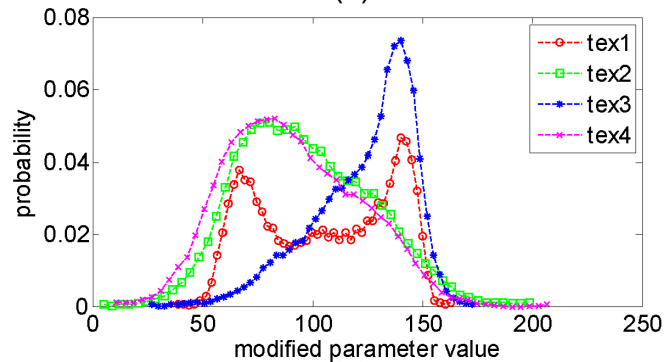

(e)

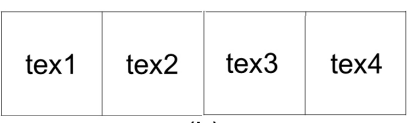

(b)

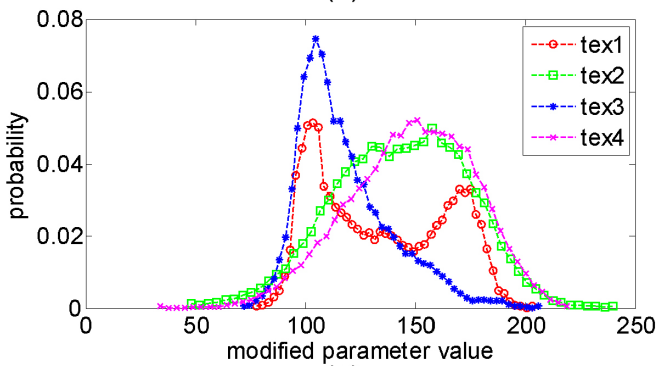

(d)

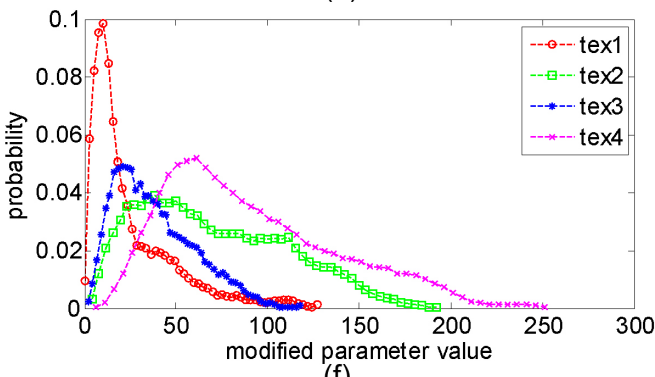

$(f)$

Figure 4: Histogram comparison. (a) image comprising four textures, (b) texture labels, and (c) intensity histograms. Histograms of spatially varying parameter images (d) histograms of $X^{h}$ (e) histograms of $X^{v}$ (f) histograms of $\sigma$ of each texture.

Table 1: Accuracy comparison with other methods. First order neighborhood system is used. bins $=50$ is used for $I B M F$ and $P L$ methods.

\begin{tabular}{cccccc}
\hline dataset & IBMF & HBMF & $P L$ & TGMRF & LBP \\
\hline BRODATZ & $88.2 \pm 1.54$ & $37.9 \pm 2.19$ & $81.0 \pm 1.13$ & $40.7 \pm 2.02$ & $89.7 \pm 1.90$ \\
OUTEX & $87.0 \pm 1.69$ & $31.7 \pm 2.19$ & $83.1 \pm 1.99$ & $40.7 \pm 2.59$ & $79.5 \pm 2.10$ \\
\hline
\end{tabular}

son. The method $P L$ is based on the spatially varying model parameters estimated using small model estimation which uses LSE (Dharmagunawardhana et al., 2012). TGMRF is the traditional GMRF feature extraction method (Manjunath and Chellappa, 1991). The method $L B P$ is based on rotational invariant uniform local binary patterns (Ojala et al., 2002) and are implemented using (Heikkila and Ahonen, 2012). Only the LBP histograms from $(\mathrm{P}=8, \mathrm{R}=1)$ are used to get roughly similar neighborhood representations as the GMRF setting where the first order neighborhood system is used.

It is clearly observed that the texture features based on spatially varying parameters significantly perform better than spatially constant parameters (table 1). Comparative studies by other authors have also reported the reduced discriminative ability of the spatially constant MRF features (Ojala et al., 2001; Hadjidemetriou et al., 2003). Note that we have only used first-order GMRFs here. By increasing the neighbor- hood size accuracies can be further improved.

Furthermore, it is observed that the IBMF has better accuracy compared to $P L$ method based on LSE (table 1). A comparison between accuracies obtained in 100 iterations from $L B P$ and $P L$ methods with $I B M F$ are shown in figure 6 . The $I B M F$ features perform better in most of the trials except for the $B R O$ $D A T Z$ dataset with $L B P$ method (figure 6a). Here, the results are comparable with $L B P$ method. However, in certain applications, for example medical image processing, when prior knowledge about pathology localization is available or smoothing priors for noise reduction is reasonably important, $I B M F$ can make use of these additional information about the problem in hand through prior distribution unlike with $L B P$.

In the present study, we have employed the local smoothing priors for $I B M F$ method. Therefore by using appropriate prior information, $I B M F$ features can perform better than the simple least square estimation based $P L$ features. 


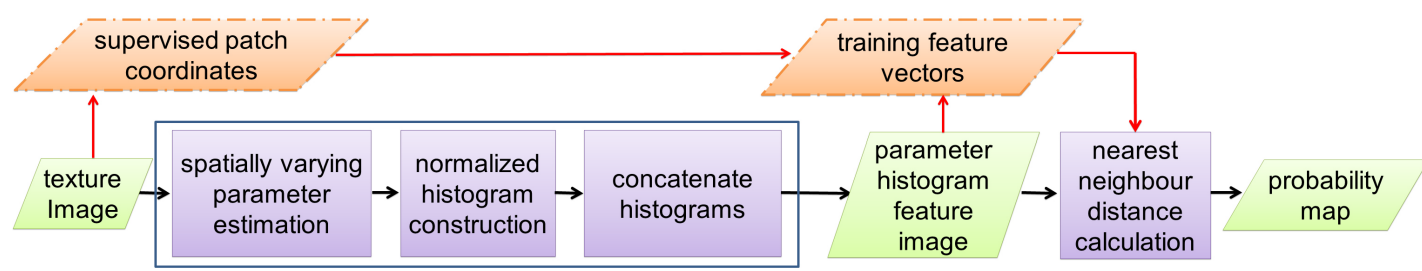

Figure 5: Supervised texture segmentation method.

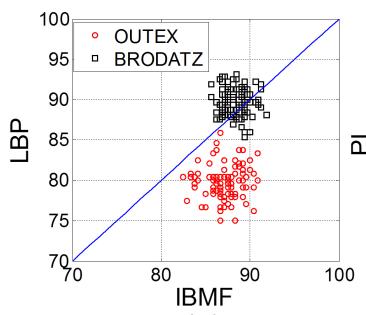

(a)

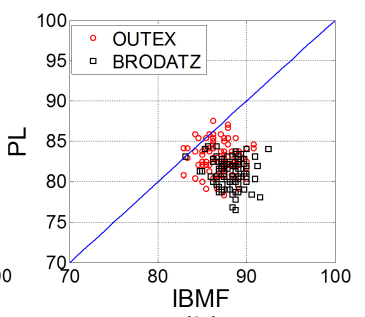

(b)
Figure 6: Comparison between accuracies obtained from $I B M F$ with $L B P$ and $P L$ in 100 trials. (a) $I B M F$ with $L B P$ (b) $I B M F$ with $P L$.

\subsection{Texture Segmentation}

We perform supervised texture segmentation using $I B M F$ features on gray scale natural images from Berkeley dataset (Martin et al., 2001) and Alpert dataset (Alpert et al., 2007). After extracting spatially varying parameters of the image, a sliding window of size $b=21 \mathrm{pxls}$ is used to construct the local parameter histograms at each pixel. The process is supervised in the sense that a supervised patch of interested texture, extracted from the input image itself, is fed into the system to calculate training data. The method is illustrated in figure 5.

First the coordinates of the supervised patch area, entered by the user, are stored and then the feature extraction for the texture image is conducted as previously discussed. Once the features are extracted for all pixels, the stored coordinates are used to select the training feature vectors from the feature image. Next, L1 norm distance between each feature vector to the nearest training feature vector is calculated. These distance measures are converted into probability values according to the following equation. Let supervised patch area containing texture of interest be $\Omega_{t} \subseteq \Omega$.

$$
p_{i}\left(\omega / \mathbf{h}_{k}, k \in \Omega_{t}\right)=\frac{\exp \left\{-D_{\min }\left(\mathbf{h}_{i}, \mathbf{h}_{k}\right)^{2}\right\}}{\max _{i \in \Omega}\left(\exp \left\{-D_{\min }\left(\mathbf{h}_{i}, \mathbf{h}_{k}\right)^{2}\right\}\right)}
$$

where local parameter histogram feature vector at a pixel $i$ is denoted by $\mathbf{h}_{i}$ and $D_{\text {min }}$ is the L1 norm distance of a feature vector to its nearest training feature vector. $p_{i}\left(\omega / \mathbf{h}_{k}, k \in \Omega_{t}\right)$ gives the probability of a pixel at location $i$ belonging to the texture of interest, $\omega$. The probability map calculated in this manner is shown in figure 7 . The probability map is then thresholded using a suitable threshold value to acquire the texture of interest (figure 7). These results are achieved using the first order neighborhood system (horizontal and vertical interaction parameters) for $I B M F$ formulation. If higher order neighborhood systems are used, the results can be further improved. However, here we mainly focus on introducing the theory of $I B M F$ and limit our interest to the first order neighborhood system.

The region boundaries obtained by thresholding the probability map gives a satisfactory texture segmentation (figure 7). Segmentation process can be further improved using other suitable advanced segmentation methods such as clustering techniques or active contours (Mahmoodi and Gunn, 2011).

\section{CONCLUSIONS}

We have proposed a novel GMRF based Bayesian texture model characterized by spatially varying model parameters for texture feature extraction. The hierarchical Bayesian formulation of the posterior model and the parameter estimation process are comprehensively explained. The distributions of estimated model parameters are used as an effective texture feature in texture classification and segmentation. The results reveal that the proposed method outperform the texture features based on spatially constant model parameters of GMRF and LSE based spatially varying parameters. It can be further concluded that in statistical model based texture feature extraction, spatially varying parameters are more suitable, capturing both spatial pixel dependencies and structural properties of the texture. The Bayesian formulation enables integration of prior knowledge to the spatially varying parameter estimation process and further improves the features based on them. Furthermore we have performed successful supervised texture segmentation on natural images to segment the areas of a given texture of interest, using probability maps and the simple thresholding technique. 

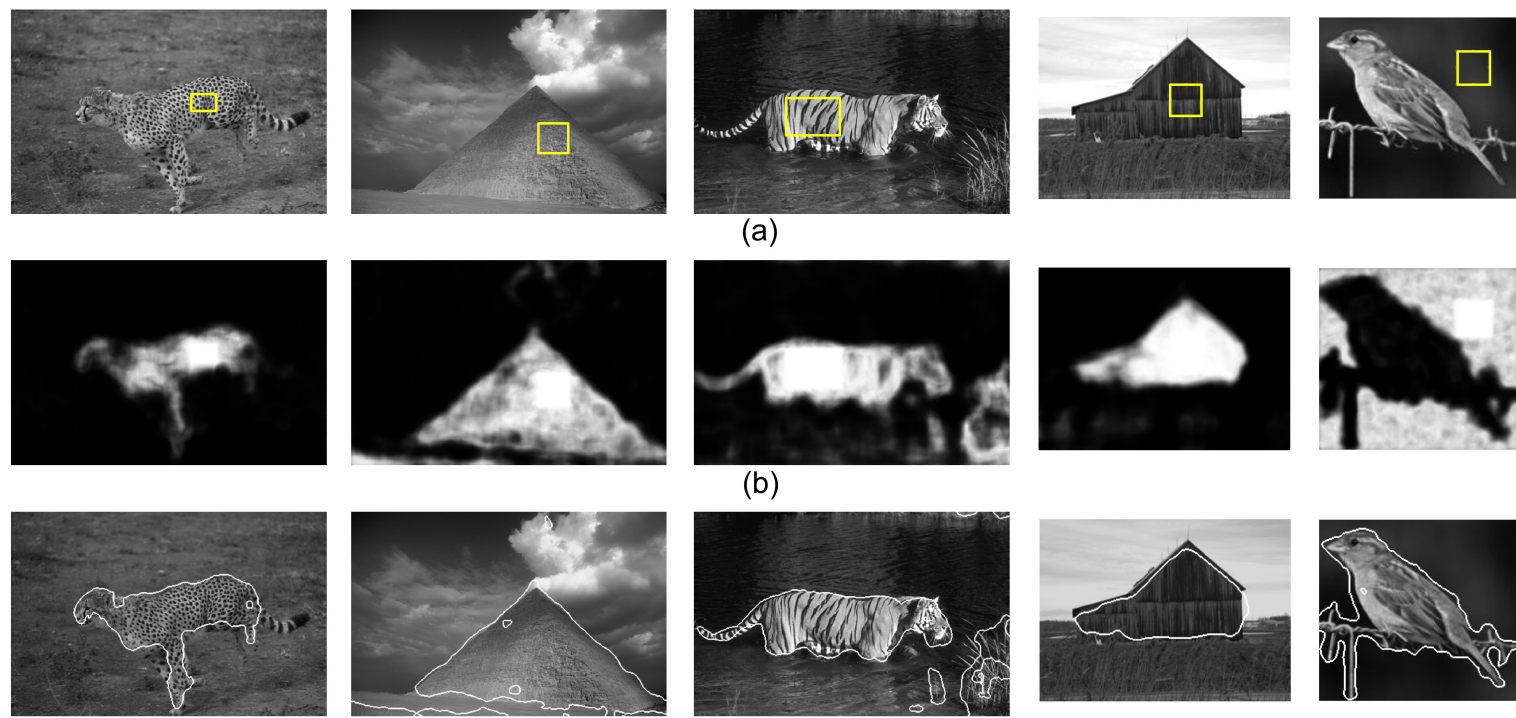

(a)
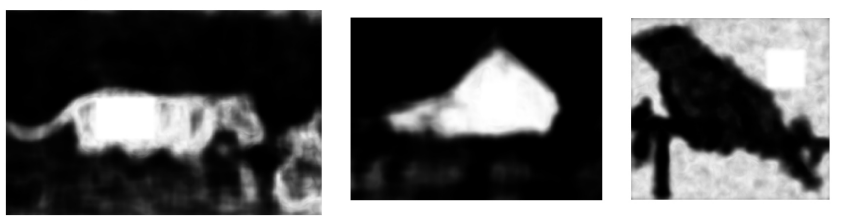

(b)
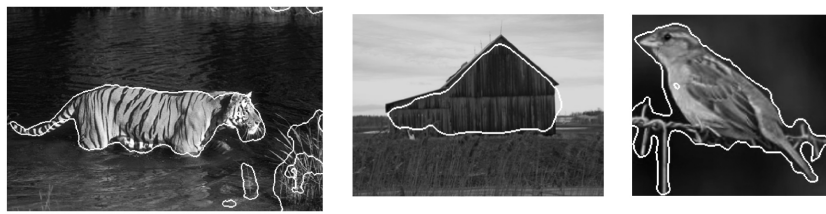

(c)

Figure 7: Supervised texture segmentation results. (a) original images with the supervised patch selected by the user. (b) probability maps. (c) boundaries obtained by thresholding the probability map. Note: first order neighborhood system is used for $I B M F$.

\section{REFERENCES}

Alpert, S., Galun, M., Basri, R., and Brandt, A. (2007). Image segmentation by probabilistic bottom-up aggregation and cue integration. In Proc. of the IEEE Conf. Computer Vision and Pattern Recognition.

Aykroyd, R. (1998). Bayesian estimation for homogeneous and inhomogeneous gaussian random fields. IEEE Trans. on pattern analysis and machine intelligence, 20:533-539.

Brodatz, P. (1996). Textures: A Photographic Album for Artists and Designers. New York: Dover.

Chellappa, R. and Chatterjee, S. (1985). Classification of textures using Gaussian Markov random fields. IEEE Trans. on Acoustics Speech and Signal Processing, 33(4):959-963.

Dharmagunawardhana, C., Mahmoodi, S., Bennett, M., and Mahesan, N. (2012). Unsupervised texture segmentation using active contours and local distributions of Gaussian Markov random field parameters. In Proc. British Machine Vision Conference, pages 88.188.11 .

Hadjidemetriou, E., Grossberg, M. D., and Nayar, S. K. (2003). Multiresolution histograms and their use for texture classification. In Int'l Workshop on Texture Analysis and Synthesis, Nice, France.

Heikkila, M. and Ahonen, T. (2012). Uniform Local Binary Patterns: Matlab code. http://www.cse.oulu.fi/CMV/Downloads/LBPMatlab.

Li, S. Z. (2009). Markov Random Field Modeling in Image Analysis. Springer-Verlag London Ltd, 3rd edn edition.

Mahmoodi, S. and Gunn, S. (2011). Snake based unsupervised texture segmentation using Gaussian Markov random field models. In Proc. 18th IEEE Int'l Conf. Image Processing, pages 1-4.

Manjunath, B. S. and Chellappa, R. (1991). Unsupervised texture segmentation using Markov random field models. IEEE Trans. on pattern analysis and machine intelligence, 13(5):478-482.

Martin, D., Fowlkes, Tal, D., and Malik, J. (2001). A database of human segmented natural images and its application to evaluating segmentation algorithms and measuring ecological statistics. In Proc. 8th Int'l Conf. Computer Vision, pages 416-423.

Ojala, T., Pietikainen, M., and Maenpaa, T. (2002). Multiresolution gray-scale and rotation invariant texture classification with local binary patterns. IEEE Trans. on pattern analysis and machine intelligence, 24:971987.

Ojala, T., Valkealahti, K., Oja, E., and Pietikäinen, M. (2001). Texture discrimination with multidimensional distributions of signed gray-level differences. Pattern Recognition, 34:727-739.

Petrou, M. and Sevilla, P. G. (2006). Image Processing, Dealing with Texture. John Wiley \& Sons Ltd.

Valkealahti, K. and Oja, E. (1998). Reduced multidimensional co-occurrence histograms in texture classification. IEEE Trans. on Pattern Analysis and Machine Intelligence, 20:90-94.

Zhao, Y., Zhang, L., Li, P., and Huang, B. (2007). Classification of high spatial resolution imagery using improved Gaussian Markov random-field-based texture features. IEEE Trans. on Geoscience and Remote Sensing, 45(5):1458-1468. 